\title{
Pancreatitis, CTCAE 5.0
}

National Cancer Institute

\section{Source}

National Cancer Institute. Pancreatitis, CT CAE 5.0. NCI Thesaurus. Code C146789.

A disorder characterized by inflammation of the pancreas with no documented pancreas

infection. 